\title{
Stability of the Solid Electrolyte Interface on the Li Electrode in Li-S Batteries
}

\author{
D. Zheng \\ Submitted to ACS Applied Materials \& Interfaces
}

April 2016

\author{
Chemistry Department
}

Brookhaven National Laboratory

\author{
U.S. Department of Energy \\ [DOE Office of Science]
}

Notice: This manuscript has been authored by employees of Brookhaven Science Associates, LLC under Contract No. DE- SC0012704 with the U.S. Department of Energy. The publisher by accepting the manuscript for publication acknowledges that the United States Government retains a non-exclusive, paid-up, irrevocable, world-wide license to publish or reproduce the published form of this manuscript, or allow others to do so, for United States Government purposes. 


\section{DISCLAIMER}

This report was prepared as an account of work sponsored by an agency of the United States Government. Neither the United States Government nor any agency thereof, nor any of their employees, nor any of their contractors, subcontractors, or their employees, makes any warranty, express or implied, or assumes any legal liability or responsibility for the accuracy, completeness, or any third party's use or the results of such use of any information, apparatus, product, or process disclosed, or represents that its use would not infringe privately owned rights. Reference herein to any specific commercial product, process, or service by trade name, trademark, manufacturer, or otherwise, does not necessarily constitute or imply its endorsement, recommendation, or favoring by the United States Government or any agency thereof or its contractors or subcontractors. The views and opinions of authors expressed herein do not necessarily state or reflect those of the United States Government or any agency thereof. 


\section{The Stability of the Solid Electrolyte Interface on the Li Electrode in Li-S Batteries}

Dong Zheng ${ }^{\dagger}$, Xiao-Qing Yang ${ }^{\ddagger}$, Deyang $Q u^{* \dagger}$

†Department of Mechanical Engineering, College of Engineering and Applied Science, University of Wisconsin Milwaukee, Milwaukee, WI 53211,USA

‡ Chemistry Department, Brookhaven National Laboratory, Upton, NY 11973,USA

*qud@uwm.edu

KEYWORDS: SEI stability, Lithium-sulfur battery, HPLC, polysulfide identification, Li sulfur reaction 


\begin{abstract}
By means of high performance liquid chromatography-mass spectroscopy, the concentration of sulfur and polysulfides was determined in non-aqueous electrolytes. The stability of sulfur and Li in eight electrolytes was studied quantitatively. It was found that sulfur reacted with Li in most of the commonly used electrolytes for lithium-sulfur batteries. The reaction products between sulfur and Li were qualitatively identified. In some cases the solid electrolyte interface on the Li can successfully prevent the interaction between $\mathrm{S}$ and $\mathrm{Li}$, however, it was found that the solid electrolyte interface was damaged by polysulfide ions.
\end{abstract}




\section{INTRODUCTION}

Rechargeable Lithium-Sulfur (Li-S) batteries are attractive due to their high theoretical energy density, $1675 \mathrm{mAh} * \mathrm{~g}^{-1} \cdot{ }^{1}$ Substantial amounts of research on Li-S batteries are focused on the material modification of the sulfur electrode to encapsulate sulfur in the nano-structure of electrodes to minimize the migration of soluble polysulfides. ${ }^{2-6}$ However, the sulfur redox reaction is considered as one of the most complex redox reactions, whose mechanisms have not been fully understood. To materialize the potential of Li-S chemistry, many technical obstacles need to be overcome, for example poor rechargeability and high self-discharge due to the "shuttle effect". Better understanding of the redox reaction mechanism of sulfur is the critical step toward a solution to the problems which hinder the practical application of Li-S chemistry. The mechanism has been studied with electrochemical and analytical methods and the mechanisms of the sulfur redox reaction were proposed. But many of them were ambiguous and sometime controversial. ${ }^{7-9}$ The major barrier to the comprehensive understanding of the sulfur reaction mechanism in a non-aqueous electrolyte is the lack of analytical method for qualitative and quantitative determination of the dissolved polysulfides. Recently, we reported the use of chromatography and mass-spectroscopy for the accurate measurements of elemental sulfur and polysulfide ions. ${ }^{10,12}$ In-situ and ex-situ techniques were used to investigate the mechanism of the sulfur reaction. ${ }^{13}$

Due to the high solubility and reactivity of polysulfide species formed in the discharge/recharge of a Li-S battery, the selection of organic electrolytes was limited. The commonly used carbonate and ester solvents for Li-ion batteries were proven unstable with the polysulfide species, the common lithium salts e.g. $\mathrm{LiBF}_{4}, \mathrm{LiPF}_{6}{ }^{1}$ also proved to be unstable. The most commonly used electrolytes for Li-S battery now are ether based electrolytes with $\mathrm{LiSO}_{3} \mathrm{CF}_{3}$ or LiTFSi. They 
were believed to be relatively stable against polysulfide species. ${ }^{1}$ However it was evident that the ether-based electrolytes still slowly react with the polysulfide species. Their long term stability is still questionable. ${ }^{1}$

The self-discharge (or the capacity loss) during the storage of a Li-S battery was reported a decade ago by Ahn et al. ${ }^{11}$ Over 20\% of capacity loss was reported after a month of storage. The self-discharge was attributed to the corrosion of the stainless-steel current collector as the following reaction:

$2 \mathrm{Li}^{+}+\mathrm{Fe}+\mathrm{nS} \longrightarrow \quad \mathrm{Li}_{2} \mathrm{~S}_{\mathrm{n}}+\mathrm{Fe}^{2+}$

And the direct reaction between $\mathrm{Li}$ anode and soluble elemental S was excluded. ${ }^{11}$ The selfdischarge of a fresh Li-S battery has seldom been studied. Recently, we noticed that the electrolyte saturated with elemental sulfur gradually changes color upon contact with Li metal. The initial clear solutions slowly changed to either green or brown solutions. In this report, the stability of Li metal with elemental $\mathrm{S}$ in various electrolytes was investigated through the identification of the remaining soluble elemental S and newly formed polysulfide ions. ${ }^{12,13}$

\section{2, Experimental}

\subsection{Chemicals}

Sulfur, Li metal, HPLC grade methanol, HPLC grade water, methyl triflate (purity over 98\%, for GC derivatization) (from Sigma Aldrich), and dimethoxyethane (DME ), lithium bis(trifluoromethane) sulfonimide (LiTFSi), lithium trifluoromethanesulfonate (LiTFS), lithium bis(oxalato) borate (LiBOB), lithium difluoro(oxalato) borate (LiDFOB), lithium tetrafluoroborate $\left(\mathrm{LiBF}_{4}\right)$, lithium hexafluorophosphate $\left(\mathrm{LiPF}_{6}\right)$, lithium perchlorate $\left(\mathrm{LiClO}_{4}\right), 1 \mathrm{M}$ 
LiTFSi in dimethoxyethane/dioxolane (DME/DOL) (battery grade from FERRO) were purchased and used without further treatment.

\subsection{Sample preparation and methods}

A Total of eight electrolytes were made, they were $1 \mathrm{M}$ LiTFS/DME, $1 \mathrm{M}$ LiTFSi/DME, $1 \mathrm{M}$ LiDFOB/DME, $1 \mathrm{M} \mathrm{LiBOB/DME,} 1 \mathrm{M} \mathrm{LiBF}_{4} / \mathrm{DME}$, 0.1 M LiPF/ $/ \mathrm{DME}, 0.1 \mathrm{M} \mathrm{LiClO} / \mathrm{DME}, 1$ M LiTFSi/DME/DOL. An excess amount of elemental sulfur was added into each electrolyte. After storing for two days, the saturated solution was taken from the clean portion of the solutions after centrifuging. $1.5 \mathrm{~mL}$ of the sulfur saturated electrolytes were then added to the sample vials with $1 \mathrm{~cm}$ diameter Li disk. The fresh surface of the Li disks was revealed with a surgical knife. All procedures were done in an argon-filled glove-box with the moisture and oxygen concentration less than $0.1 \mathrm{ppm} . \mathrm{LiNO}_{3}$ was not added in the electrolyte, because we would like to investigate the baseline reactions. The roles of $\mathrm{LiNO}_{3}$ during cycling are under investigation in our lab.

To monitor the reaction between sulfur and the electrolytes, the stock electrolytes and the electrolytes exposed to Li were sampled and analyzed at different times (1 day, 2 days, 4 days, 8 days) by HPLC. The electrolytes reacted with $\mathrm{Li}$ for 8 days were also analyzed by adding $20 \mathrm{uL}$ methyl triflate into the above mixture. All sample solutions for HPLC analysis were put into airtight HPLC sample vials before taken out of the glove-box for HPLC analysis.

A Shimadzu LC-20AD quaternary pump with a Shimadzu SIL-20A autosampler was used to deliver a methanol/water mobile phase through an Agilent Zobrax $\mathrm{C}_{18}$ column (from Agilent, $\mathrm{C}_{18}$,

4.6*50 mm, $5 \mathrm{um}$ ) at a flow rate of $0.70 \mathrm{~mL}^{*} \mathrm{~min}^{-1}$. The injection volume was $10 \mathrm{uL}$. A binary gradient of mobile phases were used to elute the injected sample out with the following condition: 
at 0 min 25\% methanol (75\% water); at 10 min 100\% methanol; at 25 min 100\% methanol; at 26 min 25\% methanol. All flow from the HPLC was introduced into the Shimadzu SPD-M20A detector, full wavelength range from $190 \mathrm{~nm}$ to $800 \mathrm{~nm}$ was recorded by Shimadzu LabSolutions Lite software, and the chromatograms discussed in this work were replotted from data at $230 \mathrm{~nm}$. Self-made standard solutions with different sulfur concentrations were used to build up the calibration curve of sulfur for quantification: the chromatographic peak areas of each standard at 14.2 min were plotted against their corresponding sulfur concentration. The concentrations of sulfur in the sample electrolytes were calculated based on the chromatographic peak areas of each sample ${ }^{12}$.

\section{RESULTS AND DISCUSSION}

Due to the nonpolar nature of elemental sulfur $\left(S_{8}\right)$, the solubility of $S_{8}$ in a non-aqueous electrolyte is relatively low compared to the solubility of polysulfide ions. For example, the saturated concentration of S in dimethyl sulfoxide (DMSO) electrolyte is around $2 \mathrm{mM},{ }^{12}$ and the concentration of polysulfide ions in a similar electrolyte can be as high as a few hundred $\mathrm{mM} .{ }^{14}$ Because of the low concentration of $S$ in the electrolyte, little attention has been paid to the potential interaction between dissolved sulfur in the electrolyte and the Li anode. Figure 1 shows an image of various S containing electrolytes after exposed to Li. The formation of polysulfide ions is clearly evident by the color change of many of the electrolytes. 


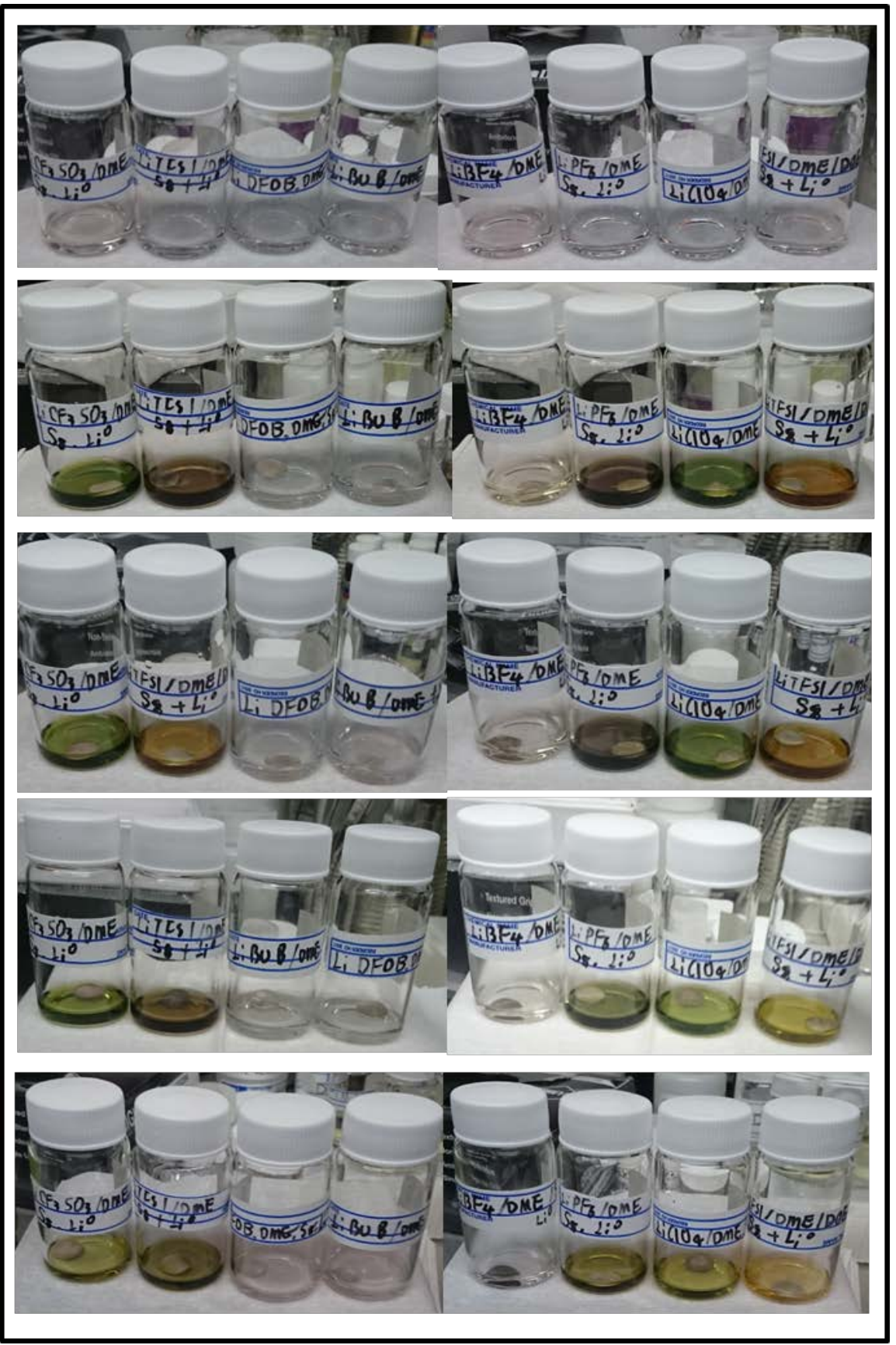

Figure 1. DME based electrolytes with elemental sulfur contacted with lithium metal at different times. From top to bottom: electrolytes with sulfur and without Li metal, electrolytes with sulfur and Li metal for 1 day, electrolytes with sulfur with Li metal for 2 days, electrolytes with sulfur with $\mathrm{Li}$ metal for 4 days, electrolytes with sulfur with Li metal for 8 days; from left to right: LiTFS/DME electrolyte, LiTFSi/DME electrolyte, LiDFOB/DME electrolyte, LiBOB/DME electrolyte, $\mathrm{LiBF}_{4} / \mathrm{DME}$ electrolyte, $\mathrm{LiPF}_{6} / \mathrm{DME}$ electrolyte, $\mathrm{LiClO}_{4} / \mathrm{DME}$ electrolyte, LiTFSi/DME/DOL electrolyte. 
To investigate the reaction between $\mathrm{S}$ and $\mathrm{Li}$, the HPLC method $^{12}$ we developed recently was used to monitor the S content in the electrolyte. Under the experimental conditions, the retention time for $\mathrm{S}_{8}$ was about $14.2 \mathrm{~min}$, the intensity of the chromatographic peak at $14.2 \mathrm{~min}$ was proportional to the concentration of the $\mathrm{S}$ in the electrolyte. Figure 2 shows the chromatograms of S containing LiTFSi/DME/DOL electrolyte and the same electrolyte after being exposed to $\mathrm{Li}$ for 8 days. The consumption of $\mathrm{S}$ in the electrolyte with $\mathrm{Li}$ was clearly demonstrated by the noticeable difference in the intensities of the sulfur peak at $14.2 \mathrm{~min}$ between the two samples.

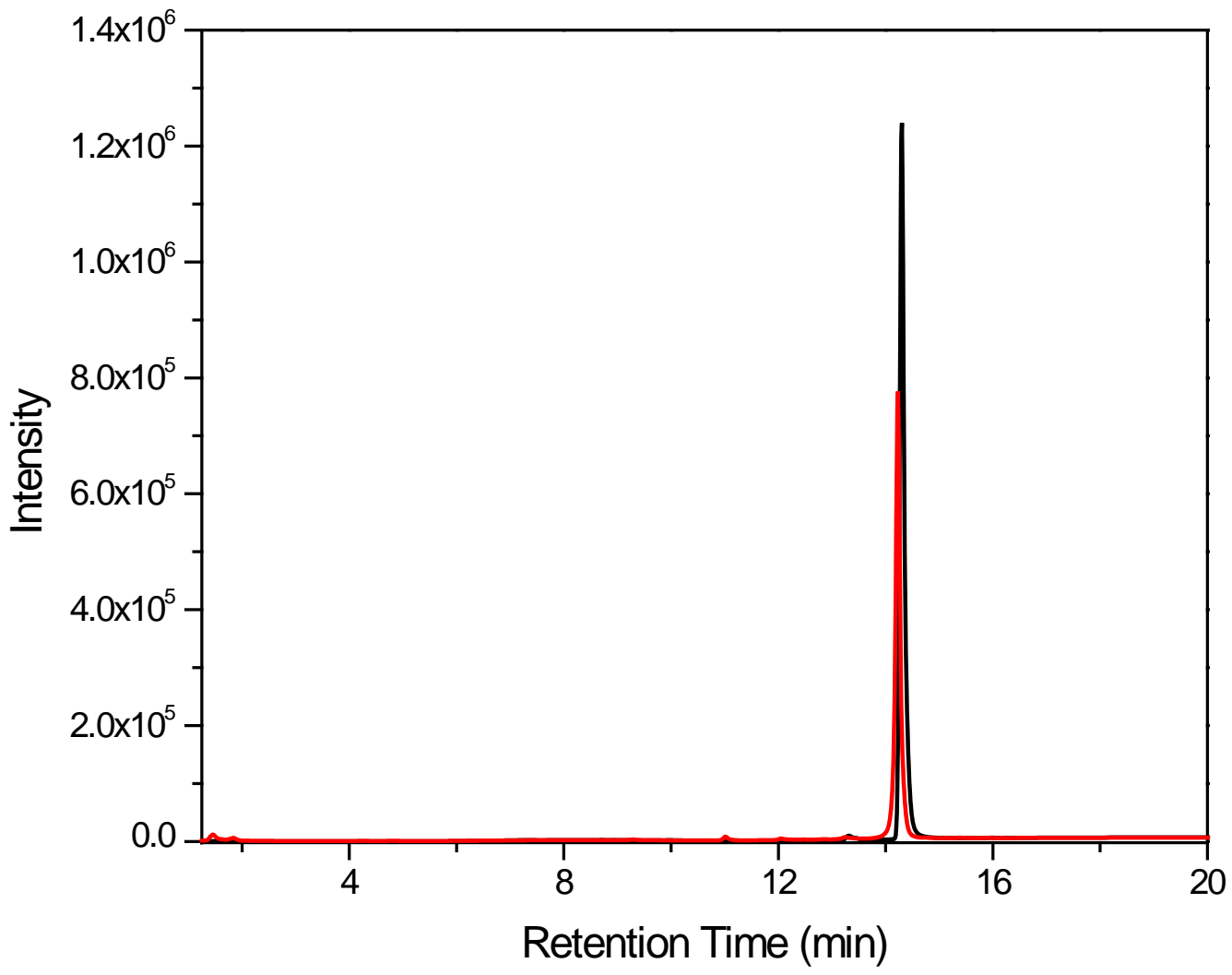

Figure 2. The chromatograms of LiTFSi/DME/DOL stock electrolyte without Li metal (in black), LiTFSi/DME/DOL stock electrolyte with Li metal for 8 days without derivatization (in red).

Figure 3 shows the changes of the remaining amount of $S$ in the electrolytes versus the time in contact with Li. Apparently, the reactivity of the dissolved S with Li varied in different 
electrolytes. Among the eight DME based electrolytes, the reaction between S and Li was greatly suppressed if not totally inhibited when LiDFOB and LiBOB salts were used. The remaining amount

of $\mathrm{S}$ in those two electrolytes hardly changed after 8 days in contact with $\mathrm{Li}$, which was consistent with the visual observations shown in figure 1 where the colours of the two electrolytes remained unchanged. While in all other six DME based electrolytes, the soluble sulfur reacted with lithium metal, the dissolved S in the electrolyte containing LiTFSi was almost completely consumed.

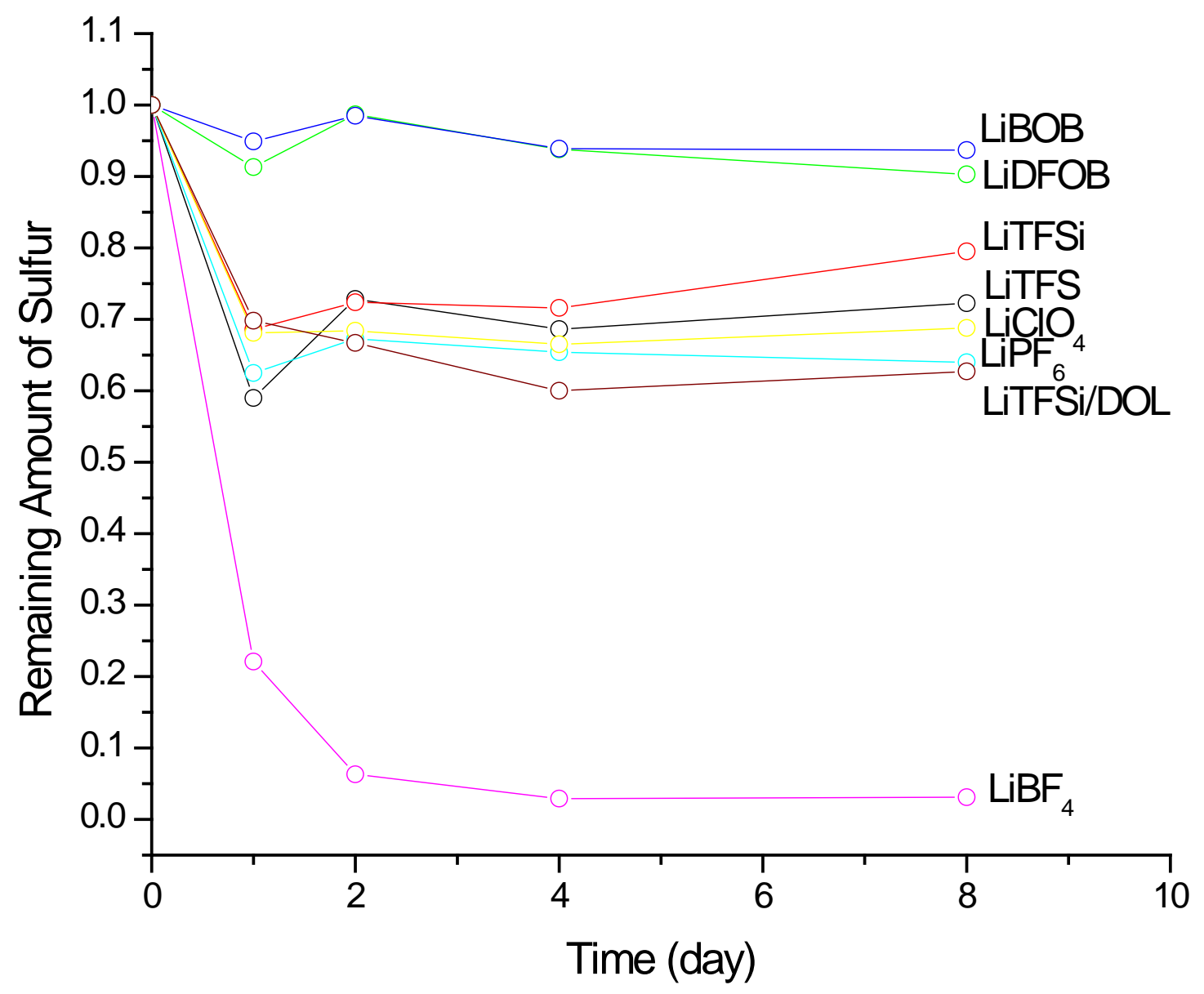

Figure 3. The changes of the remaining amount of sulfur in DME based electrolytes with elemental sulfur contacted with lithium metal at different time. For the remaining amount of 
sulfur, 1 means that the amount of sulfur in electrolyte has no change before and after contact with lithium metal; and 0 means that the amount of sulfur in electrolyte is undetectable after contact with lithium metal (all samples were analyzed by HPLC without derivatization). LiTFS/DME electrolyte in black, LiTFSi/DME electrolyte in red, LiDFOB/DME electrolyte in green, $\mathrm{LiBOB} / \mathrm{DME}$ electrolyte in blue, $\mathrm{LiBF}_{4} / \mathrm{DME}$ electrolyte in magenta, $\mathrm{LiPF}_{6} / \mathrm{DME}$ electrolyte in cyan, $\mathrm{LiClO}_{4} / \mathrm{DME}$ electrolyte yellow, LiTFSi/DME/DOL electrolyte in wine.

From figure 3, it appears that a stable solid electrolyte interface (SEI) layer was formed on some of the Li discs which prevented further reaction with $\mathrm{S}$, due to the amount of S remaining stable after the initial decrease. We will prove that this conclusion was premature and the stable SEI layers did not form. This example exemplifies the importance of proper experimental design and the risk of rushing to a conclusion.

The sample analysis by HPLC in figure 3 was performed without derivatization, the polysulfide species could potentially decompose and S forms in the disproportionation in the water and methanol solution, which was the mobile phase of the LC. To eliminate the potential influence of such reactions, methyl triflate was used to derivatize the polysulfides in the samples before injecting into the HPLC. The polysulfides were stabilized and the potential reactions in the mobile phase were thus avoided. Methyl triflate can efficiently react with polysulfide anions and form dimethyl polysulfide species through the following reaction. ${ }^{15}$

$\mathrm{S}_{(\mathrm{n})}^{2-}+2 \mathrm{CH}_{3} \mathrm{SO}_{3} \mathrm{CF}_{3} \longrightarrow\left(\mathrm{CH}_{3}\right)_{2} \mathrm{~S}_{(\mathrm{n})}+2 \mathrm{CF}_{3} \mathrm{SO}_{3}^{-}$

It has been demonstrated that dimethyl polysulfide species are stable in the HPLC mobile phase condition and the elemental sulfur has no reaction with methyl triflate. ${ }^{13,15}$

Figure 4 shows the remaining amount of $\mathrm{S}$ in the electrolytes after eight days of exposure to Li. The polysulfides were derivatized with methyl triflate. Clearly, except in the electrolytes 
containing LiDFOB and LiBOB salts, S reacted with Li in every other DME electrolyte. No effective SEI layer was evident since almost all the $\mathrm{S}$ was consumed. It is not surprising that the disproportionation reaction occurred in the LC mobile phase, which resulted in the difference between the results shown in figure 3 and figure 4.

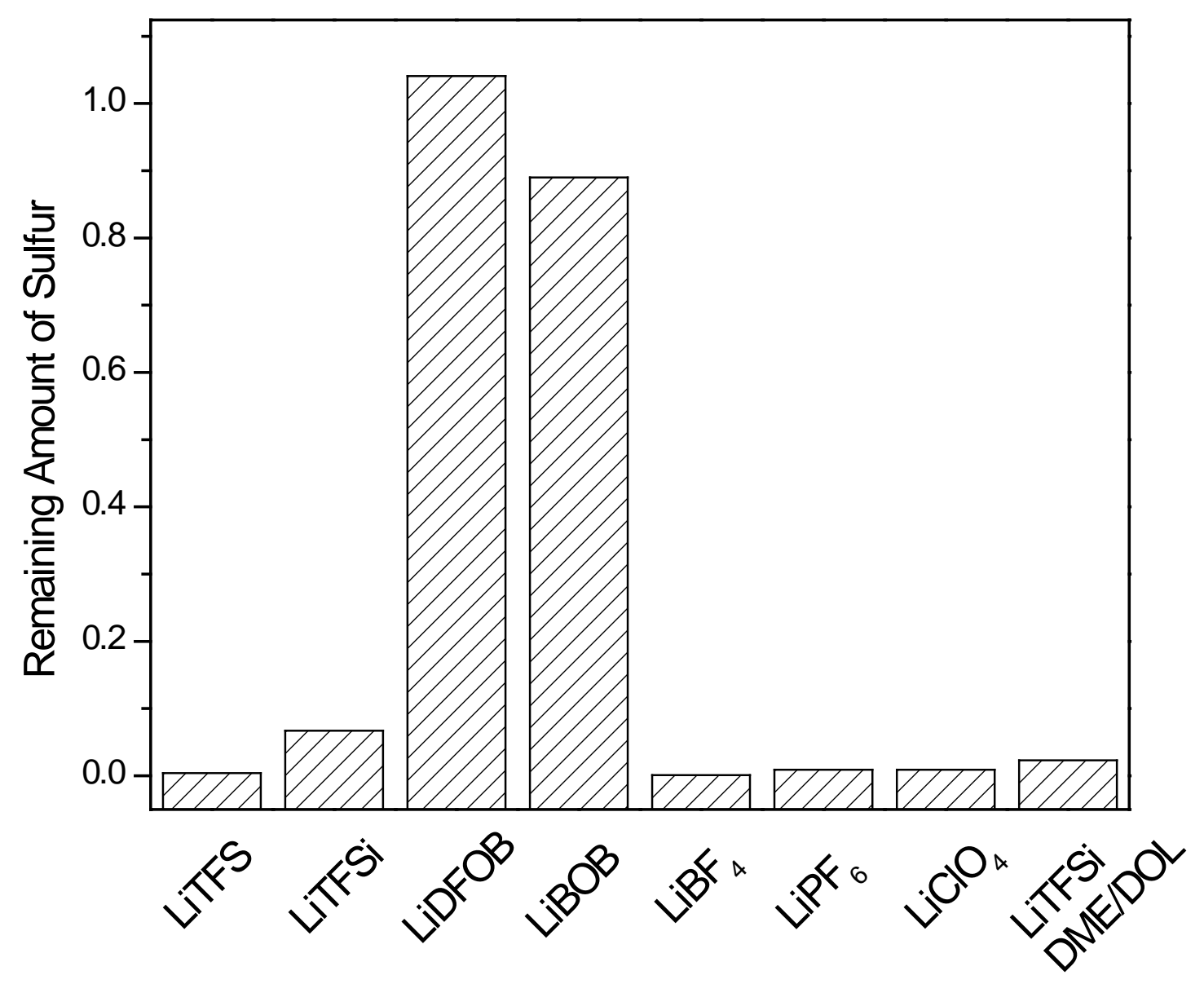

Figure 4. The changes of the remaining amount of sulfur in DME based electrolytes with elemental sulfur contacted with lithium metal at different times. For the remaining amount of sulfur, 1 means that the amount of sulfur in electrolyte has no change before and after contact with lithium metal; and 0 means that the amount of sulfur in electrolyte is undetectable after contact with lithium metal (all samples were analyzed by HPLC after derivatization with methyl triflate). 
Since S reacts with metallic Li forming polysulfides, then it was of great interest in identifying the polysulfide ions formed during the reaction. The S dissolved in the most commonly used electrolyte (LiTFSi/DME/DOL) in Li-S batteries was exposed to Li for 8 days, the solution was then derivatized and analyzed by chromatogram. The results are shown in figure 5.Multiple peaks appear between 9 and $17.5 \mathrm{~min}$, the sulfur peak appears at $14.2 \mathrm{~min}$. The polysulfide ions eluting at different time were determined by atmospheric pressure chemical ionization mass spectroscopy (APCI-MS). The examples of MS for the peaks at about 11 and 12 min are shown as insets in figure 5. The detailed analysis for the methylated derivatization was reported in ref. 13. The assignments of each peak are tabulated in the table 1 .

Table 1. The assignment of chromatographic peaks for methylated polysulfide species and sulfur.

\begin{tabular}{|c|c|}
\hline Chemicals & $\begin{array}{c}\text { Elution time } \\
\text { (min) }\end{array}$ \\
\hline$\left(\mathrm{CH}_{3}\right)_{2} \mathrm{~S}_{3}$ & 9.23 \\
\hline$\left(\mathrm{CH}_{3}\right)_{2} \mathrm{~S}_{4}$ & 10.99 \\
\hline$\left(\mathrm{CH}_{3}\right)_{2} \mathrm{~S}_{5}$ & 12.09 \\
\hline$\left(\mathrm{CH}_{3}\right)_{2} \mathrm{~S}_{6}$ & 12.90 \\
\hline$\left(\mathrm{CH}_{3}\right)_{2} \mathrm{~S}_{7}$ & 13.57 \\
\hline $\mathrm{S}_{8}$ & 14.23 \\
\hline
\end{tabular}

For the other electrolytes, such as LiTFS/DME, LiTFSi/DME, $\mathrm{LiPF}_{6} / \mathrm{DME}$, and $\mathrm{LiClO}_{4} / \mathrm{DME}$, the chromatograms for the derivatized electrolytes (after contact with lithium metal for 8 days) were similar to the chromatograms of LiTFSi/DME/DOL electrolyte shown in figure 5, but the distributions of dimethyl polysulfides were different. However the chromatograms of LiDFOB/DME, $\mathrm{LiBOB} / \mathrm{DME}$, and $\mathrm{LiBF}_{4} / \mathrm{DME}$ electrolytes were substantially different from those five electrolytes. Figure 6 shows the chromatogram of LiDFOB/DME and LiBOB/DME electrolytes, the only major chromatographic peak is the elemental S at 14.2 min. No peaks of 
dimethyl polysulfides can be seen in chromatogram figure 6 . This observation confirmed the stability of sulfur and Li in LiDFOB/DME and LiBOB/DME electrolytes.

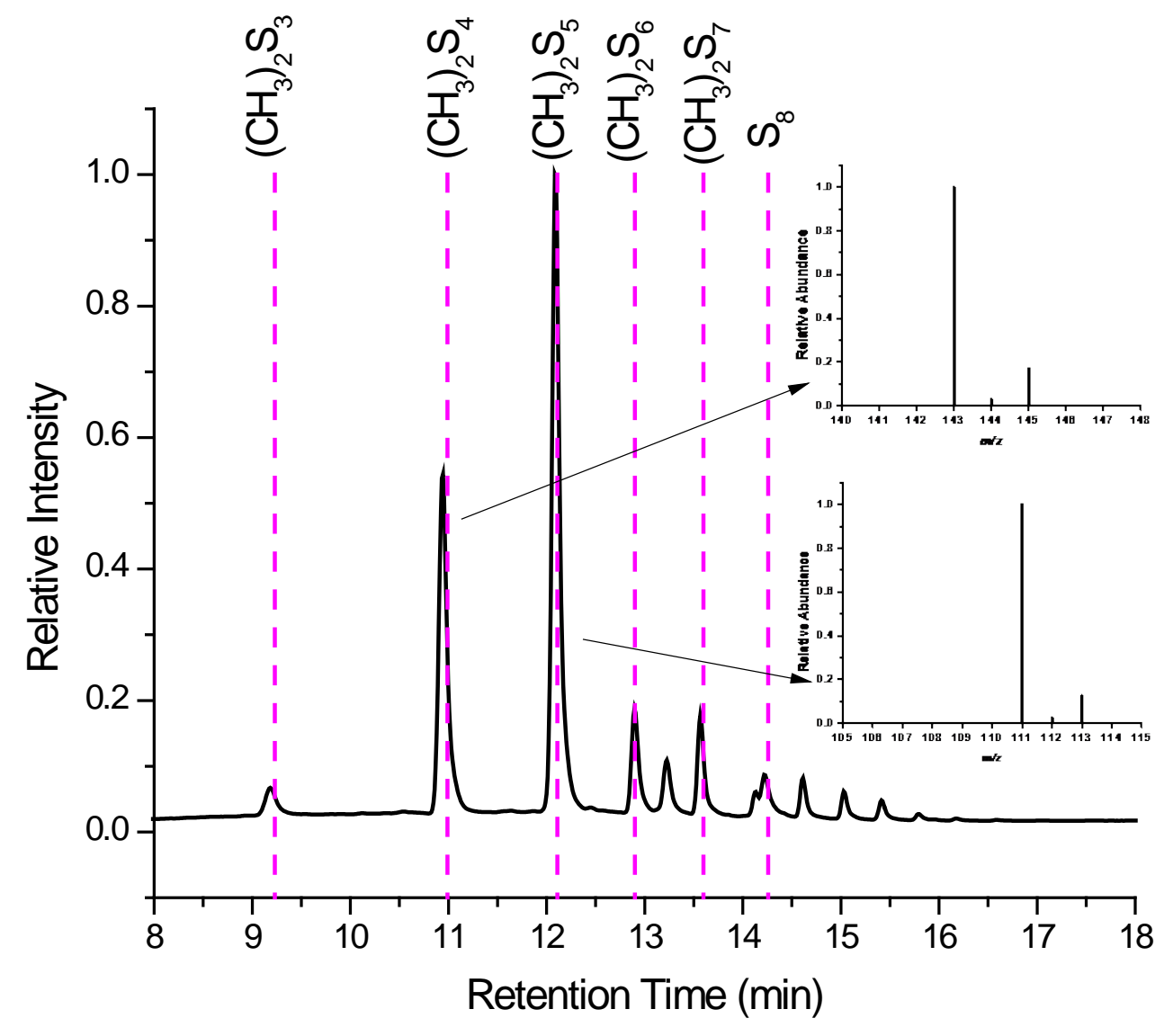

Figure 5. The chromatogram of LiTFSi/DME/DOL electrolyte with Li metal for 8 days then derivatized by methyl triflate. The insect spectra are the corresponding MS spectra for $\left(\mathrm{CH}_{3}\right)_{2} \mathrm{~S}_{4}$ and $\left(\mathrm{CH}_{3}\right)_{2} \mathrm{~S}_{5}$.

It's well known that once in contact with an organic electrolyte, an SEI layer would form on the surface of a Li electrode to prevent any further reaction with the electrolyte. ${ }^{16}$ Apparently, the stability of the SEI layer on Li anode in DME based electrolyte containing S is highly dependent on the salts used. For example, by using XPS analysis Xu et al ${ }^{17}$ found that the surface chemical species on the graphitic anode in LiBOB electrolyte were greatly different from those in $\mathrm{LiPF}_{6}$ electrolyte with the same carbonate mixture as a solvent. To further demonstrate the importance 
of the SEI layer, we mixed $\mathrm{Li}_{2} \mathrm{O}$, which was believed as one of the major components in the SEI layer in an ether based electrolyte, with sulfur containing 1M LiTFSi/DME/DOL electrolyte. After 8 days of storage in a glove box, the electrolyte was derivatized by methyl triflate and analyzed by HPLC. The chromatogram of the HPLC analysis was shown in figure 7. Dimethyl polysulfide peaks are clearly shown in figure 7 demonstrating the reaction between $\mathrm{Li}_{2} \mathrm{O}$ and $\mathrm{S}$. Clearly, $\mathrm{S}$ reacts not only with $\mathrm{Li}$ metal but also reacts with the SEI components e.g. $\mathrm{Li}_{2} \mathrm{O}$, which would destroy the SEI layer. Probably the moisture in the electrolyte (although extremely low) is the key reason, since with moisture the $\mathrm{Li}_{2} \mathrm{O}$ could form $\mathrm{LiOH}$, which is known to react with sulfur.

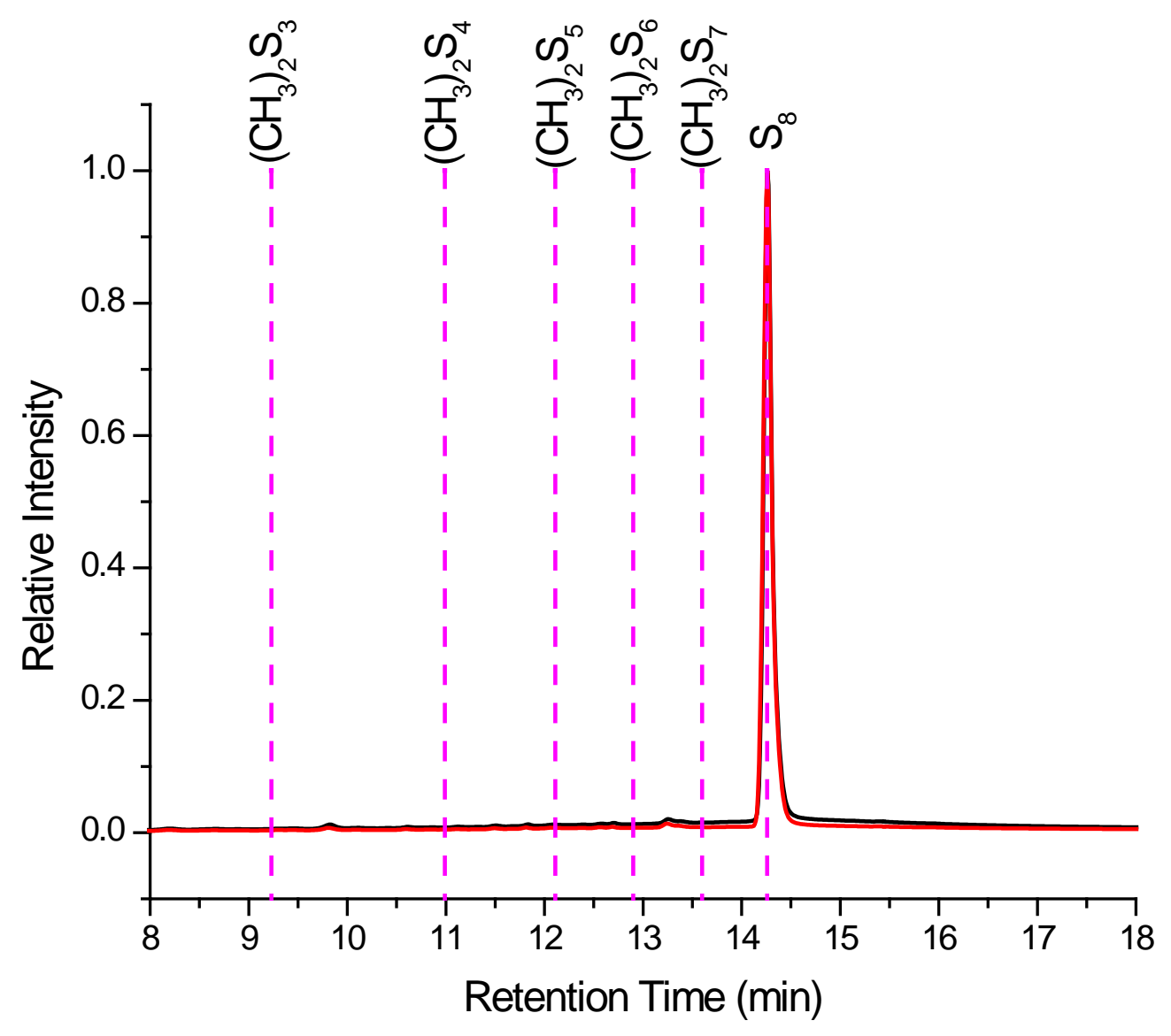


Figure 6. The chromatograms of LiBOB/DME electrolyte with $\mathrm{Li}$ metal (in black) and LiDFOB/DME electrolyte with Li metal (in red) for 8 days then derivatized by methyl triflate.

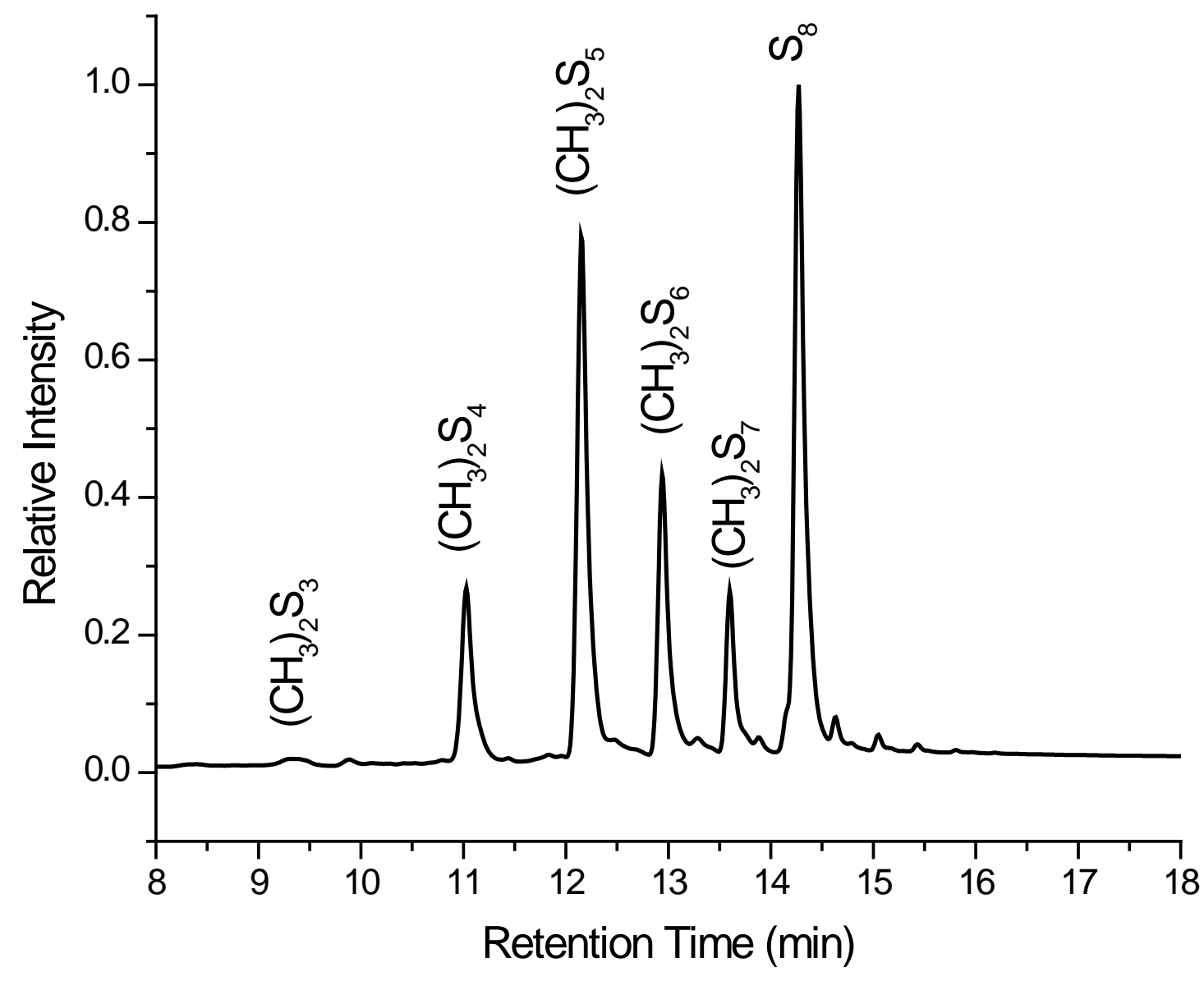

Figure 7. The chromatogram of the LiTFS/DME electrolyte with elemental sulfur after mixed with $\mathrm{Li}_{2} \mathrm{O}$ for 8 days. The electrolyte was analyzed by HPLC after derivatization with methyl triflate.

Figure 8 shows HPLC results of the derivatized $\mathrm{LiBF}_{4} / \mathrm{DME}$ electrolyte after stored with $\mathrm{S}$ and Li for eight days. No peak is observable in figure 8, which means all the S was consumed, but no polysulfides ions could be detected either. The result was consistent with what was reported in ref. 1. The polysulfide species were consumed by $\mathrm{LiBF}_{4}$ through reaction 3:

$\mathrm{LiBF}_{4}+\mathrm{Li}_{2} \mathrm{~S}_{\mathrm{n}} \longrightarrow \mathrm{LiBS}_{\mathrm{n}} \mathrm{F}_{2}+2 \mathrm{LiF}$ 


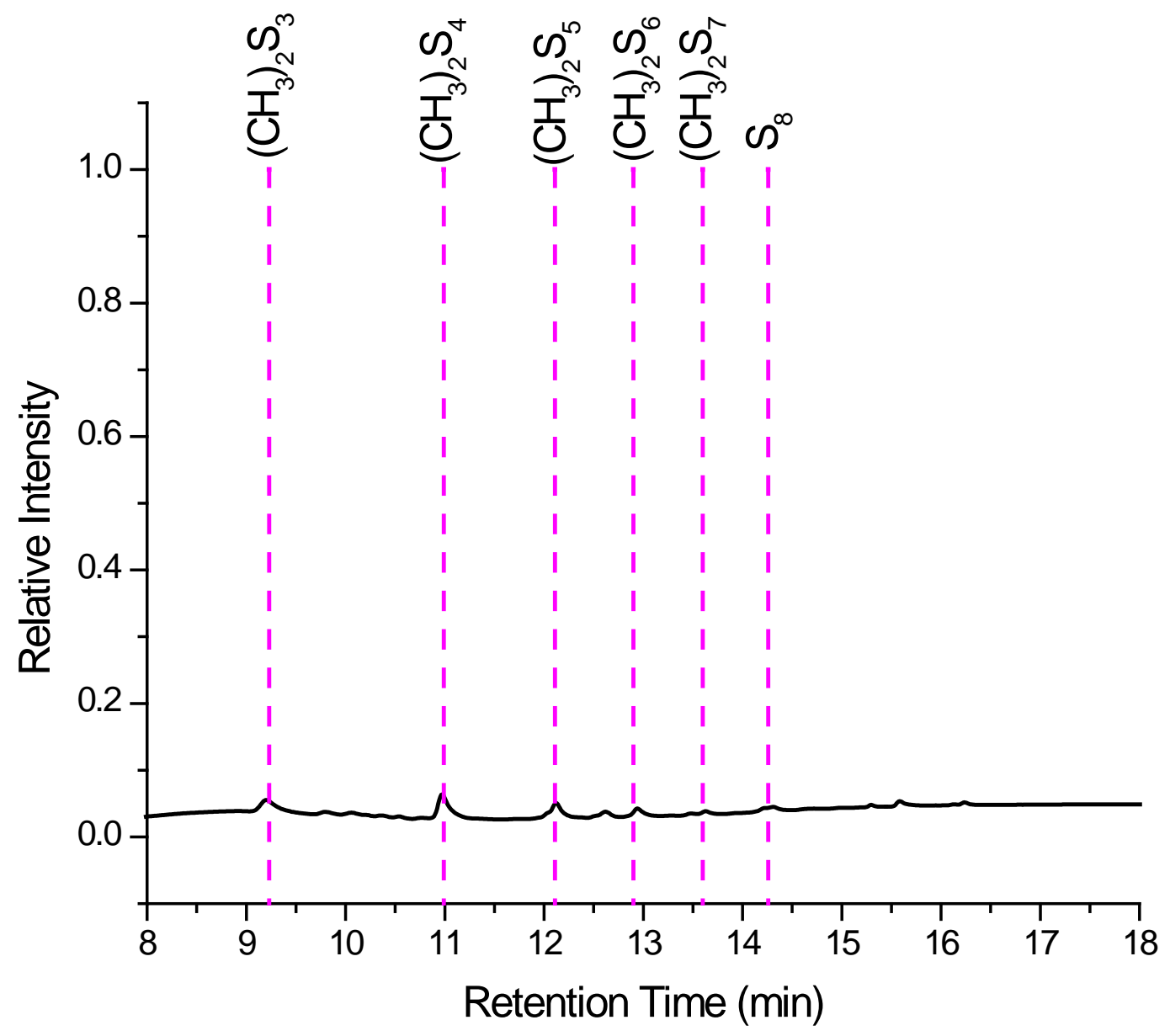

Figure 8. The chromatogram of $\mathrm{LiBF}_{4} / \mathrm{DME}$ electrolyte with Li metal for 8 days then derivatized by methyl triflate.

Although $\mathrm{S}$ and $\mathrm{Li}$ were proven stable in both $\mathrm{LiDFOB} / \mathrm{DME}$ and LiBOB/DME electrolytes, whether they are suitable for Li-S batteries would also depend on their chemical stability with

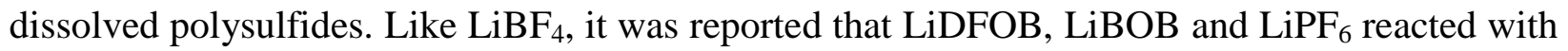
polysulfide species formed during discharge of Li-S batteries. ${ }^{1}$ To investigate the compatibility of both LiDFOB and LiBOB DME electrolytes with polysulfide species, a polysulfide solution made by mixing $\mathrm{S}$ with $\mathrm{Li}_{2} \mathrm{~S}$ in electrolyte of $0.1 \mathrm{M}$ LiTFS/DME was studied. The $0.1 \mathrm{M}$ LiTFS 
solution was used to mimic the actual electrolyte environment. LiTFS (20 mM), LiBOB (20 $\mathrm{mM})$, and LiDFOB (20 mM)were then added/mixed with the baseline polysulfide solution in separate container. After storing for one day, the polysulfide solutions were taken out, derivatized with methyl triflate and analyzed by HPLC. The chromatographic results are summarized in figure 9. Figure 9 (A) shows the chromatogram of the derivatized original polysulfide solution, the distribution of polysulfide species in this original polysulfide solution was considered as the baseline. Figure 9B demonstrates that there was no substantial change of the distribution of polysulfide ions after adding $20 \mathrm{mM}$ of LiTFS, therefore any effects due to the dilution or the change of ionic strength can be ignored. It is clearly shown in figure 9C that LiBOB reacted with the polysulfide ions in the electrolyte. The distribution of the sulfur compounds in the electrolyte changed significantly. It is interesting that it seems an oxidation reaction occurred since the majority of polysulfides became S eventually. Additional work has to be done to fully understand the chemical reaction between polysulfides and LiBOB, but we hypothesis the reaction as shown in reaction 4

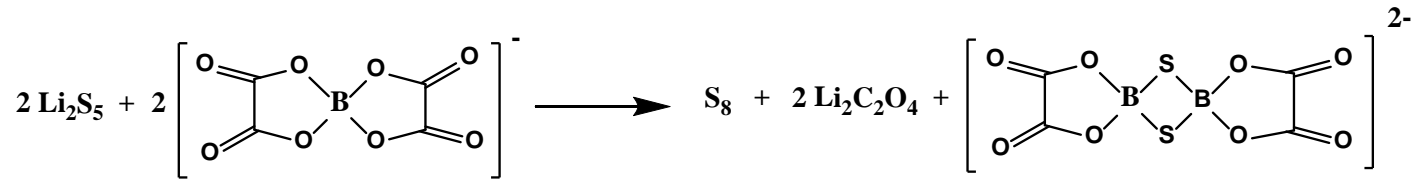

Figure 9D shows the distribution of sulfur compounds after the addition of LiDFOB. Although the change was not as momentous as that in figure 9C, a notable shift of the distribution toward longer chain polysulfides and S can be observed.

It is worth mentioning that the quantitative and qualitative analysis of sulphur compounds in the electrolyte is critical for the investigation of Li-S. Electrochemical cycling may not provide enough information, due to the slow kinetics of the reaction. Good performance at high rate may 
not imply equally good performance at low rate. The some chemical interactions are slow, so are the diffusion of dissolved polysulfide ions. Gao et al. investigated a large numbers of electrolytes in combination with various solvents and salts. They concluded that salts did not have any significant impact on the sulphur redox reaction. ${ }^{18}$ Clearly, salts in the electrolyte of a Li-S cells do play important roles.

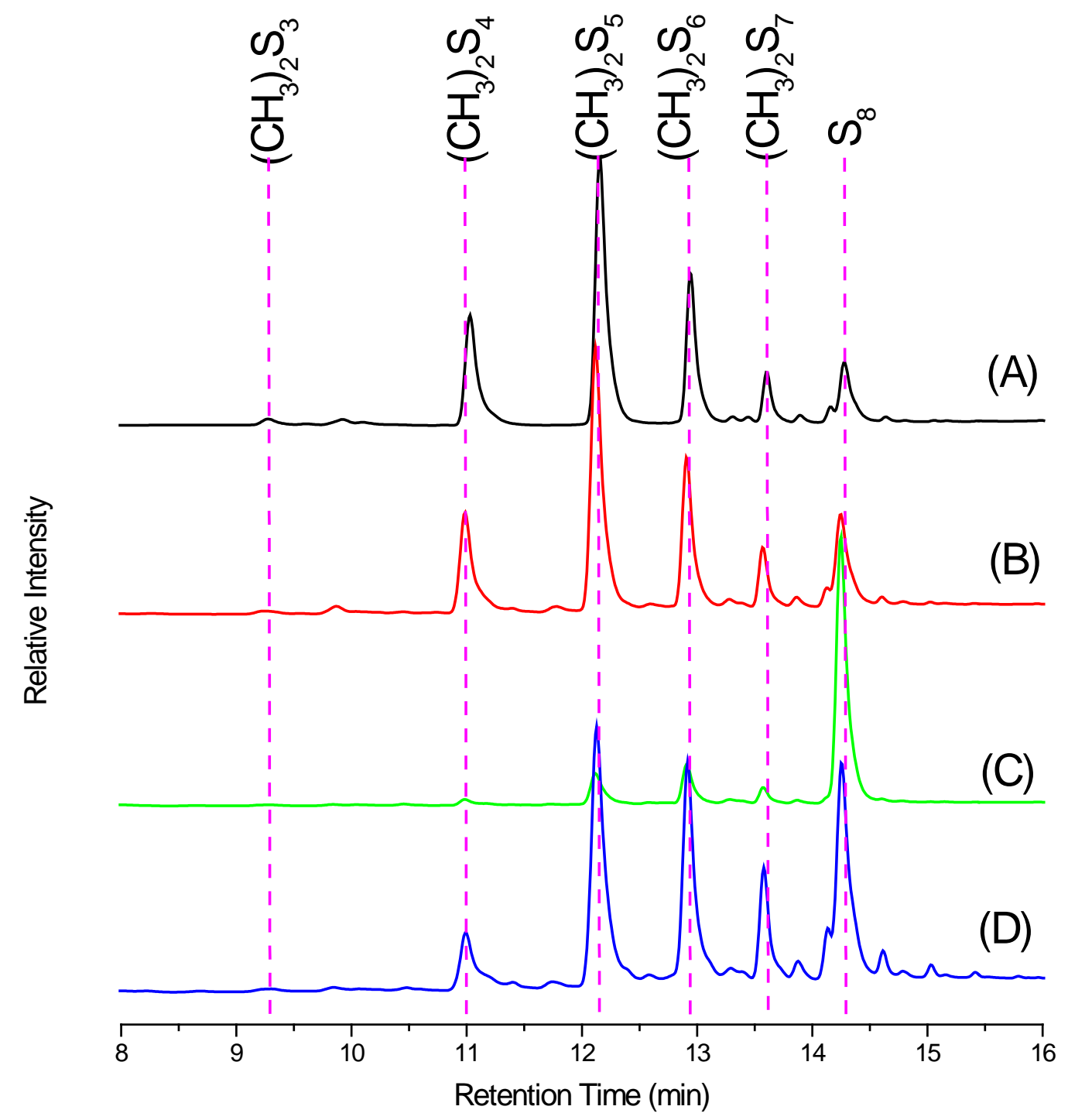

Figure 9. The selective chromatograms of polysulfide mixtures in $0.1 \mathrm{M}$ LiTFS/DME electrolytes: (A), original polysulfide mixture; (B), mixed with LiTFS (20 mM); (C); mixed with 
LiBOB (20 mM); (D), mixed with LiDFOB (20 mM). All samples were first derivatized by methyl triflate before HPLC analysis.

Conclusively, the homogeneous and heterogeneous chemical interaction of sulfur compounds with both lithium salts and lithium metal are very complex. A perfect electrolyte for Li-S chemistry has not been found yet. The HPLC-MS essays we developed for the quantitative and qualitative determination of all dissolved sulfur and polysulfide species have proved critical for the investigation and development of Li-S electrolyte systems.

\section{CONCLUSION}

Various commonly used electrolytes for Li-S batteries were studied using HPLC with a derivatization method. Unfortunately, none of them were found very suitable for Li-S batteries. Because either the dissolve $\mathrm{S}$ destroyed the SEI layer and reacted with the $\mathrm{Li}$, such as in LiTFS/DME, LiTFSi/DME, or LiTFSi/DME/DOL; or the salts reacts with polysulfide ions, e.g. LiDFOB/DME and LiBOB/DME.

The new HPLC MS essay we developed previously provides an effective analytical means to quantitatively and qualitatively determine the dissolved sulfur and polysulfide ions in an electrolyte. It is the minimum requirements for a Li-S battery electrolyte that the SEI layer is strong enough to prevent the interaction of $\mathrm{Li}$ with $\mathrm{S}$ and polysulfides, and that the salts are stable in the electrolyte with dissolved S and polysulfides species. 


\section{AUTHOR INFORMATION}

\section{Corresponding Author}

* D.Y. Qu. E-mail: qud@uwm.edu

\section{ACKNOWLEDGMENT}

The authors from UWM and BNL are indebted to the Assistant Secretary for Energy Efficiency and Renewable Energy, Office of Vehicle Technologies, under the program of Vehicle Technology Program, under Contract Number DE-SC0012704. 


\section{REFERENCES}

(1) Zhang, S.S. Liquid Electrolyte Lithium/Sulfur Battery: Fundamental Chemistry, Problems, and Solutions. J. Power Sources, 2013, 231, 153-162.

(2) Ji, X.L.; Lee, K.T.; Nazar, L.F. A Highly Ordered Nanostructured Carbon-Sulphur Cathode for Lithium-Sulphur Batteries. Nat. Mater., 2009, 8, 500-506.

(3) Zhang, B.; Qin, X.; Li, G.R.; Gao, X.P. Enhancement of Long Stability of Sulfur Cathode by Encapsulating Sulfur into Micropores of Carbon Spheres. Energy Environ. Sci., 2010, 3, 15311537

(4) Guo, J.C.; Xu, Y.H.; Wang, C.S. Sulfur-Impregnated Disordered Carbon Nanotubes Cathode for Lithium-Sulfur Batteries. Nano Lett., 2011, 11, 4288-4294.

(5) Zhang, G.Y.; Yang, Y.; Cha, J.J.; Hong, S.S.; Cui, Y. Hollow Carbon NanofiberEncapsulated Sulfur Cathodes for High Specific Capacity Rechargeable Lithium Batteries. Nano Lett., 2011, 11, 4462-4467.

(6) Zheng, S.Y.; Chen, Y.; Xu, Y.H.; Yi, F.; Zhu, Y.J.; Liu, Y.H.; Yang, J.H.; Wang, C.S. In Situ Formed Lithium Sulfide/Microporous Carbon Cathodes for Lithium-Ion Batteries. ACS Nano, 2013, 7, 10995-11003.

(7) Leghie, P.; Lelieur, J.-P.; Levillain, E. Comments on the Mechanism of the Electrochemical Reduction of Sulphur in Dimethylformamide. Electrochem. Commun., 2002, 4, 406-411.

(8) Manan, N.S.A.; Aldous, L.; Alias, Y.; Murray, P.; Yellowlees, L.J.; Lagunas, M.C.; Hardacre, C. Electrochemistry of Sulfur and Polysulfides in Ionic Liquids. J. Phys. Chem. B, 2011, 115, 13873-13879.

(9) Jung, Y.; Kim, S.; Kim, B.-S.; Han, D.-H; Park, S.-M.; Kwak, J. Effect of Organic Solvents and Electrode Materials on Electrochemical Reduction of Sulfur. Int. J. Electrochem. Sci., 2008, 3, 566-577.

(10) Zheng, D.; Qu, D.Y..; Yang, X.-Q.; Yu, X.Q.; Lee, H.S.; Qu, D.Y. Quantitative and Qualitative Determination of Polysulfide Species in the Electrolyte of Lithium-Sulfur Battery 
Using HPLC ESI/MS with One-Step Derivatization. Adv. Energy Mater., 2015, DOI:19.1002/aenm.201401888.

(11) Ryu, H.S.; Ahn, H.J.; Kim, K.W.; Ahn, J.H.; Lee, J.Y.; Cairns, E.J. Self-Discharge of Lithium-Sulfur Cells Using Stainless-Steel Current-Collectors. J. Power Sources, 2005, 140, 365-369.

(12) Zheng, D.; Zhang, X.R.; Li, C.; McKinnon, M.E.; Sadok, R.G.; Qu, D.Y.; Yu, X.Q.; Lee, H.S.; Yang, X.-Q.; Qu, D.Y. Quantitative Chromatographic Determination of Dissolved Elemental Sulfur in the Non-Aqueous Electrolyte for Lithium-Sulfur Batteries. J. Electrochem. Soc., 2015, 162, A203-A206.

(13) Zheng, D.; Zhang, X.R.; Wang, J.K.; Qu, D.Y.; Yang, X.-Q.; Qu, D.Y. Reduction Mechanism of Sulfur in Lithium-Sulfur Battery: From Elemental Sulfur to Polysulfide. J. Power Sources, 2016, 301, 312-316.

(14) Rauh, R.D.; Shuker, F.S.; Marston, J.M.; Brummer, S.B. Formation of Lithium Polysulfides in Aprotic media. J. Inorg. Nucl. Chem., 1977, 39, 1761-1766.

(15) Kamyshny, A.; Goifman, A.; Gun, J.; Rizkov, D.;Lev, O. Equilibrium Distribution of Polysulfide Ions in Aqueous Solutions at $25^{\circ} \mathrm{C}$ : A New Approach for the Study of Polysulfides' Equilibria. Environ. Sci. Technol., 2004, 38, 6633-6644.

(16) Xu, K. Nonaqueous Liquid Electrolytes for Lithium-Based Rechargeable Batteries. Chem. Rev., 2004, 104, 4303-4417.

(17) Xu, K.; Lee, U.; Zhang, S.S.; Wood, M.; Jow, T.R. Chemical Analysis of Graphite/Electrolyte Interface Formed in LiBOB-Based Electrolytes. Electrochem. Solid-State Lett., 2003, 6, A144-A148.

(18) Gao, J.; Lowe, M.A.; Kiya, Y.; Abruna, H.D. Effects of Liquid Electrolytes on the ChargeDischarge Performance of Rechargeable Lithium/Sulfur Batteries: Electrochemical and in-Situ X-ray Absorption Spectroscopic Studies. J. Phys. Chem. C, 2011, 115, 25132-25137 


\section{TABLE OF CONTENTS GRAPHIC AND SYNOPSIS}

The stability of sulfur and Li in different ether-based electrolytes was studied quantitatively by HPLC. In most of the commonly used electrolytes for lithium-sulfur batteries, sulfur was consumed by reacting with Li. Some reaction products between sulfur and Li were qualitatively identified. In some electrolytes the solid electrolyte interface on the Li can successfully prevent the interaction between $\mathrm{S}$ and $\mathrm{Li}$, however, It was found that the solid electrolyte interface was damaged by polysulfide ions.

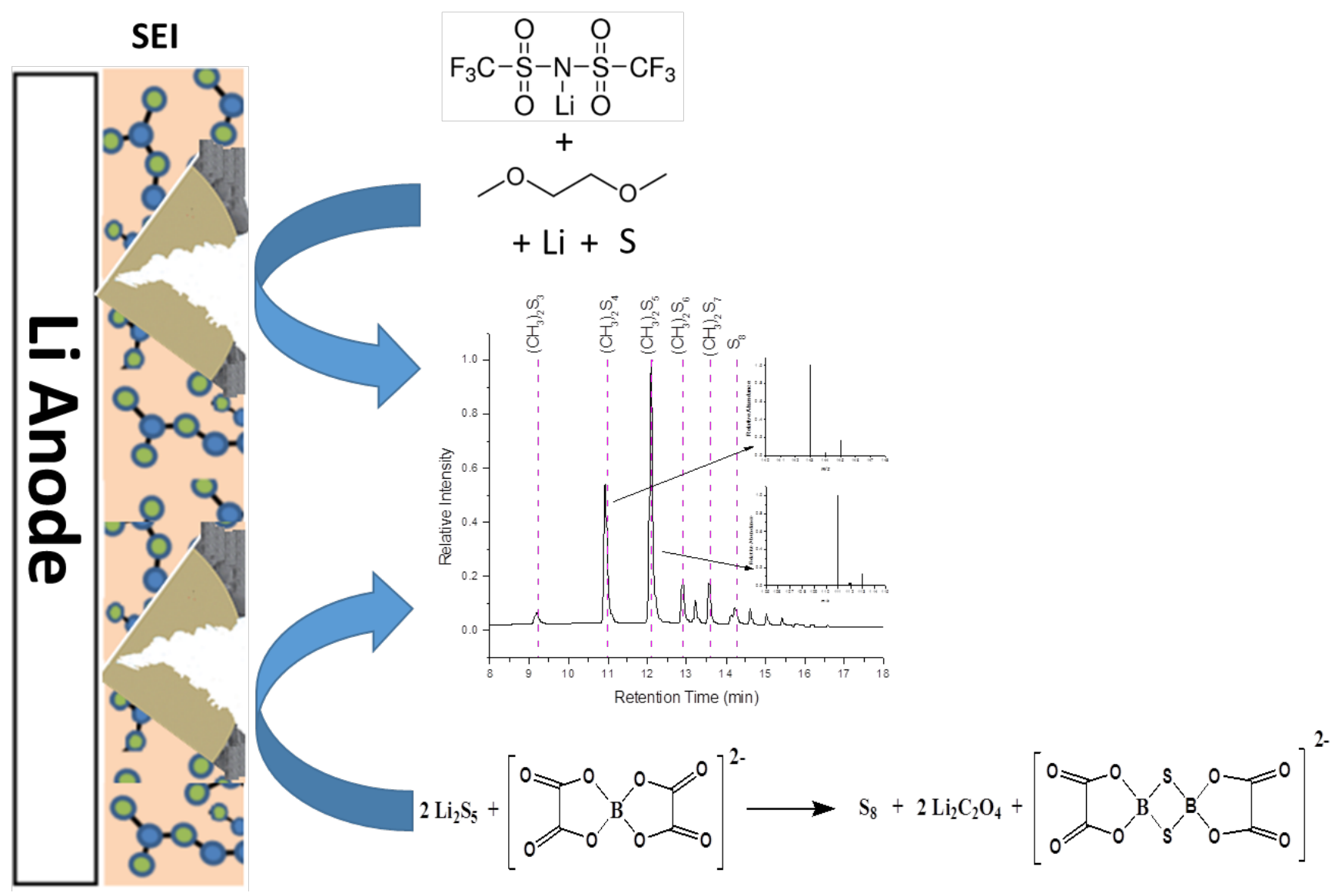

